\title{
Suttonella indologenes
}

National Cancer Institute

\section{Source}

National Cancer Institute. Suttonella indologenes. NCI Thesaurus. Code C86816.

A species of aerobic, Gram negative, rod shaped bacteria assigned to the phylum

Proteobacteria. This species is alkaline phosphatase and oxidase positive, catalase

negative and is unable to produce acid from mannitol or sorbitol. S. indologenes is an

opportunistic pathogen that has been reported to cause eye infections and prosthetic valve endocarditis in immunocompromised patients. 